\title{
C-QUALITY: cost and quality-of-life pharmacoeconomic analysis of antidepressants used in major depressive disorder in the regional Italian settings of Veneto and Sardinia
}

\author{
This article was published in the following Dove Press journal: \\ ClinicoEconomics and Outcomes Research \\ 2 December 2013 \\ Number of times this article has been viewed
}

\author{
Claudio Mencacci' \\ Eugenio Aguglia ${ }^{2}$ \\ Giovanni Biggio 3 \\ Lodovico Cappellari ${ }^{4}$ \\ Guido Di Sciascio ${ }^{5}$ \\ Andrea Fagiolini ${ }^{6}$ \\ Giuseppe Maina ${ }^{7}$ \\ Alfonso Tortorella ${ }^{8}$ \\ Pablo Katz ${ }^{9}$ \\ Claudio Ripellino9 \\ 'Department of Mental Health, \\ Fatebenefratelli Hospital, Milan, \\ ${ }^{2}$ Department of Clinical and \\ Molecular Biomedicine, University \\ of Catania, Catania, ${ }^{3}$ Department of \\ Experimental Biology and Center \\ of Excellence for the Neurobiology \\ of Drug Dependence, University of \\ Cagliari, Calgiari, ${ }^{4}$ Department of \\ Mental Health, Camposampiero \\ Hospital, Padova, ${ }^{5}$ Department of \\ Psychiatry, Policlinico Hospital, Bari, \\ ${ }^{6}$ Department of Molecular Medicine, \\ University of Siena and Department \\ of Mental Health, University of Siena \\ Medical Center, Siena, ${ }^{7}$ Department of \\ Neurosciences, University of Torino, \\ Torino, ${ }^{8}$ Department of Mental Health, \\ University of Naples SUN, Naples, \\ ${ }^{9}$ CSD Medical Research Srl, Milan, Italy
}

Correspondence: Claudio Ripellino

CSD Medical Research Srl,

Viale E. Jenner, 53 - 20159 Milano, Italy

Tel +390269770279

Fax +390269010325

Email claudio.ripellino@cegedim.com
Background: Major depression is a commonly occurring, seriously impairing, and often recurrent mental disorder. Selective serotonin reuptake inhibitors (SSRIs) and serotoninnorepinephrine reuptake inhibitors (SNRIs) are the treatments most commonly used for major depressive disorder. The objective of this study was to assess the cost-effectiveness of SSRIs and SNRIs in the treatment of major depressive disorder in two Italian regional settings, ie, Veneto and Sardinia.

Methods: A decision analytic model was adapted from the Swedish Dental and Pharmaceutical Benefits Agency to reflect current clinical practice in the treatment of major depressive disorder in the most significant Italian regions. This adaptation was possible as a result of collaboration with an expert panel of Italian psychiatrists and health economists. The population comprised patients with a first diagnosis of major depressive disorder and initiating one SSRI or SNRI drug for the first time. The time frame used was 12 months. Efficacy and utility data for the model were retrieved from the literature and validated by the expert panel. Local data were used for resource utilization and for treatment costs based on the perspective of each regional health service. Scenario analyses and probabilistic sensitivity analyses were performed to test the robustness of the model.

Results: Base case analysis showed that escitalopram is associated with the largest health gain (in quality-adjusted life years) and a lower total cost at one year for Sardinia (except for sertraline, against which it was cost-effective) and for Veneto, and therefore dominates the other treatment strategies, given that more quality-adjusted life years are achieved at a lower total cost. Scenario analyses and probabilistic sensitivity analyses support the robustness of the model.

Conclusion: The results indicate that escitalopram is the most cost-effective pharmacologic treatment strategy for both regional health services compared with all SSRIs and all SNRIs used in the first-line treatment of major depressive disorder.

Keywords: antidepressants, major depressive disorder, cost-effectiveness quality of life, Italy

\section{Introduction}

Major depressive disorder (MDD) is a commonly occurring heterogeneous disorder with a highly variable course, an inconsistent response to treatment, and no established physiopathologic mechanism. ${ }^{1}$ The World Health Organization ranked MDD as the principal cause of years lost due to disability and the third cause of disability worldwide, projecting that by 2030 it will be the first leading cause. ${ }^{2}$ Globally, MDD affects around 150 million adults, and in Italy the number of people with the disease is estimated to be about 5 million with a lifelong prevalence between $8 \%$ and $13 \%{ }^{3}$ 
MDD is considered an important burden in terms of direct costs, ${ }^{4}$ which represent $31 \%$ of the total costs, which are paid by patients, their families, and the health care services, and also in terms of indirect costs, which account for $62 \%$ of the overall costs of depression. ${ }^{5,6}$ In 2004, the worldwide economic burden of the disease was estimated at USD 83.1 billion. $^{7}$

MDD is associated with an increased risk of relapse after a first episode and a higher risk of suicidal behavior. Depressive disorders impact society mainly by increasing suicide risk; in a study of 102 fatal suicides, almost $70 \%$ of victims had had an affective disorder. ${ }^{8}$ Depressive disorders also have a major impact on quality of life. In a study of quality of life impairment in depressive disorders, $63 \%$ of respondents with MDD had severely impaired quality of life, while $56 \%$ of those with dysthymia and globally $85 \%$ of those with double depression (MDD and dysthymia) have been reported to have quality of life impairment in the severe range. ${ }^{9}$

The main therapeutic alternatives for MDD include antidepressant medication, psychotherapy, and neuromodulatory strategies. Selective serotonin reuptake inhibitors (SSRIs) and serotonin-norepinephrine reuptake inhibitors (SNRIs) are two classes of antidepressants with a better safety profile than the traditional drugs, such as the tricyclic antidepressants. ${ }^{10}$

A recent literature review by Cipriani et $\mathrm{al}^{11}$ identified differences in terms of both efficacy and acceptability among commonly prescribed antidepressants in favor of escitalopram and sertraline. In particular, this meta-analysis showed that venlafaxine, escitalopram, mirtazapine, and sertraline were more effective in terms of response than duloxetine, paroxetine, reboxetine, fluoxetine, and fluvoxamine. In terms of acceptability, escitalopram, citalopram, bupropion, and sertraline had better performance than other secondgeneration antidepressants. Another review by Cipriani et al ${ }^{12}$ identified some statistically significant differences favoring escitalopram over other antidepressive agents for acutephase treatment of major depression in terms of efficacy (citalopram and fluoxetine) and acceptability (duloxetine). However, there was insufficient evidence to detect a difference between escitalopram and other antidepressants in early response to treatment.

Another paper by Aguglia et $\mathrm{al}^{13}$ reported that use of SSRIs increased from $7.5 \%$ (2003) to $13.1 \%$ (2009) while the utilization of SNRIs increased from $0.8 \%$ to $2.5 \%$. The most important increase over the 6-year period was described for escitalopram (+2.78\%). A higher persistence in therapy was reported for SSRIs versus SNRIs (15.1\% versus 13.0\%), and escitalopram was associated with the highest percentage of persistent patients and the highest number of days of uninterrupted therapy. Overall, around 10\% of antidepressant users switched their first choice during one year of follow-up, while escitalopram was associated with the highest frequency of "high" adherers (28.5\%). ${ }^{13}$

The objective of the present study, called C-QUALITY (Cost and Quality of Life Pharmacoeconomic Analysis on MDD in Italy), was to assess the cost-effectiveness of SSRI and SNRI drugs used in first-line treatment of MDD, adopting Italian Regional Health Service perspectives. In this paper, we describe the cases of Veneto and Sardinia. The Italian National Health Service (Servizio Sanitario Nazionale) is a complex multitier system, involving three different levels of government, ie, central (Ministry of Health), regional (Assessorati alla Salute), and local (Aziende Sanitarie Locali [ASLs]). The Ministry of Health is responsible for national planning and coordination of regional activities, in order to guarantee the same essential health care (Livelli Essenziali di Assistenza [LEA]) in all areas of the country. Regions are competent to define their own health plans so as to organize delivery of services within their own territory in accordance with central government planning. The local ASLs have administrative and financial autonomy and are directly involved in producing and commissioning services in the geographic area under their responsibility. Due to regional autonomy, each of them can decide, always in accordance with the national level and respecting LEA guidelines, the administrative characteristics of their health service in terms of prices of the different health services provided (examinations, visits, hospitalizations) and cost-sharing rules (copayment, ticket). For these reasons, Veneto and Sardinia have been analyzed because of their widely different health service costs (examinations, visits, hospitalizations) and cost-sharing rules (copayment, ticket) for depression.

\section{Materials and methods Description of the model}

An expert panel was organized to evaluate a pharmacoeconomic model developed by the Swedish Dental and Pharmaceutical Benefits Agency (TLV) $)^{14,15}$ and to adapt it to Italian clinical practice. The panel comprised eight psychiatrists from different Italian regions and two health economists from an independent agency. Starting from the TLV model, a decision analytic model was developed to simulate the management of Italian patients with MDD over a time horizon of 12 months. Main inputs and parameters of the TLV model, such as decision tree, remission probabilities, and utilities, were discussed by the expert panel and adapted to the Italian context, with inputs related to costs retrieved as local data. 
This pharmacoeconomic analysis involved patients with a first diagnosis of MDD and receiving an SSRI (escitalopram, fluvoxamine, fluoxetine, paroxetine, citalopram, sertraline) or SNRI (duloxetine, venlafaxine) antidepressant for the first time. The decision tree was developed using TreeAge Pro 2011 software (TreeAge Software Inc, Williamstown, MA, USA) and its structure is presented in Figure 1.

The treatment objective was to achieve remission defined by a score of $\leq 7$ on the Hamilton Depression Rating Scale ${ }^{17}$ or $\leq 12$ on the Montgomery Asberg Depression Rating Scale. ${ }^{18}$ Once in remission, if the patient did not relapse (defined as a new major depressive episode occurring within 6 months after remission), then she/he was treated with maintenance treatment for 6 months in accordance with international treatment guidelines; the local expert panel agreed with this treatment pattern. If the patient relapsed, it was assumed that relapse occurred within 4 months of the start of treatment. Patients who did not respond, defined as not achieving remission on first-line treatment, moved to a second-line therapy; a proportion of these patients might attempt suicide. Those patients who achieved remission in the second step of treatment received maintenance treatment for 6 months, whereas those who did not achieve remission on second-line treatment moved to the third step of treatment. Patients moved to the third and fourth treatment steps according to the same criteria described for the second step of treatment.

\section{Model parameters}

\section{Efficacy}

For the first line of treatment, remission probabilities were estimated from an independent meta-analysis conducted by the TLV as specified in Table 1. More than 80 studies were included in this meta-analysis for around 20,000 patients. ${ }^{14}$ Further, the authors of the meta-analysis had applied adjustments in order to exclude potential bias related to level of sponsorship of the studies. The expert panel adapted the second, third, and fourth pharmacologic treatment lines described in the STAR $* D^{16}$ (Sequenced Treatment Alternatives to Relieve Depression) study to standard Italian clinical practice.

If the initial treatment line failed, patients moved to a second treatment line that could be a switching strategy or a combination strategy; if the second treatment line failed, patients passed to a third treatment line as an augmentation strategy and, finally, if this last strategy also failed, patients continued to a fourth treatment line comprising a switching strategy carried out during hospitalization (Table 2).

Regarding second, third, and fourth treatment lines, the expert panel agreed to adapt STAR*D remission probabilities to the treatments not considered in the STAR*D study, but usually administered in normal Italian clinical practice. Remission probabilities of these treatments were assessed as the mean value of each drug class considered in the STAR *D study and Mencacci et al ${ }^{15}$ (Table 3). It was assumed that the risk of relapse would be $11 \%$ and that relapse would occur after 4 months. ${ }^{19}$ For suicide attempts and the probability of dying in such an attempt, a probability value of 0.031 and 0.1 , respectively, was set following the studies by Löthgren and $\mathrm{Khan}^{20,21}$ and the work by Mencacci et al ${ }^{15}$ (Table 4).

\section{Costs}

Considering the perspective of the model, this analysis took into account direct medical costs (drugs, patient

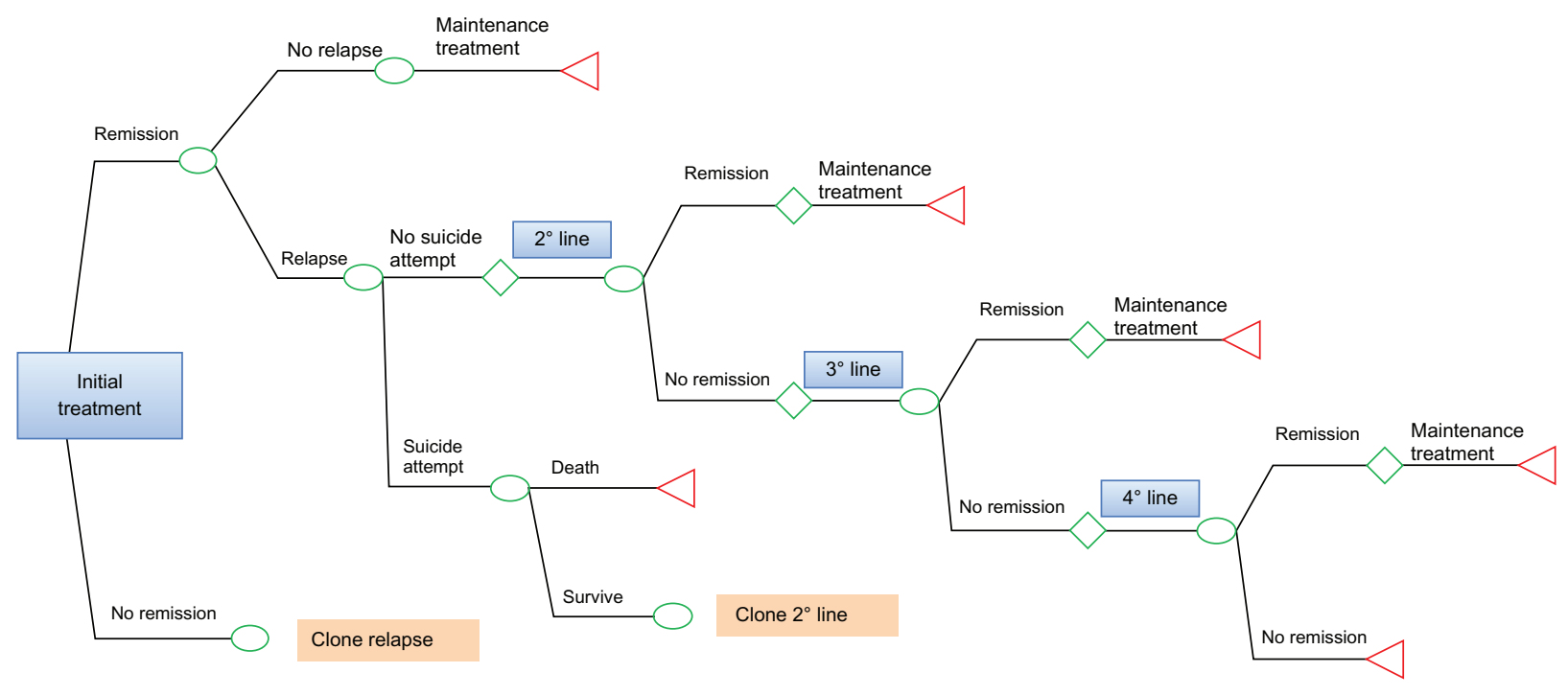

Figure I Decision analytic model. 
Table 5 Pharmaceutical costs and resource utilization

\begin{tabular}{|c|c|c|c|c|}
\hline \multirow{2}{*}{$\begin{array}{l}\text { Pharmaceutical costs per month } \\
\text { Drug }\end{array}$} & \multicolumn{2}{|l|}{ Veneto } & \multicolumn{2}{|l|}{ Sardinia } \\
\hline & Assumed DDD & Assumed MD & Assumed DDD & Assumed MD \\
\hline Citalopram & $€ 5.38$ & $€ 10.76$ & $€ 6.75$ & $€|3.5|$ \\
\hline Escitalopram & $€ 22.99$ & $€ 34.49$ & $€ 24.12$ & $€ 36.19$ \\
\hline Fluoxetine & $€ 4.49$ & $€ 8.99$ & $€ 6.41$ & $€ 12.82$ \\
\hline Fluvoxamine & $€ 8.90$ & $€ 17.80$ & $€ 10.85$ & $€ 21.70$ \\
\hline Paroxetine & $€ 7.99$ & $€ 15.98$ & $€ 9.52$ & $€ 19.03$ \\
\hline Sertraline & $€ 4.56$ & $€|| .4 \mid$ & $€ 5.86$ & $€ 14.66$ \\
\hline Duloxetine & $€ 33.63$ & $€ 50.45$ & $€ 37.61$ & $€ 56.42$ \\
\hline Venlafaxine XR & $€ 10.96$ & $€ 16.44$ & $€ \mid 4.24$ & $€ 21.36$ \\
\hline Amitriptyline & $€ 0.76$ & $€ 1.40$ & $€ 2.12$ & $€ 3.88$ \\
\hline Bupropion & $€ 34.48$ & $€ 25.86$ & $€ 35.78$ & $€ 26.84$ \\
\hline Reboxetine & $€ 23.52$ & $€ 29.39$ & $€ 26.12$ & $€ 32.64$ \\
\hline Mirtazapine & $€ 14.17$ & $€ 14.17$ & $€ 15.54$ & $€ 15.54$ \\
\hline Lithium carbonate & $€ 0.59$ & $€ 0.79$ & $€ \mathrm{I} .44$ & $€ 1.92$ \\
\hline Thyroid agents (T3) & $€ 3.88$ & $€ 1.29$ & $€ 5.49$ & $€ 1.83$ \\
\hline Quetiapine & $€ 63.75$ & $€ 47.81$ & $€ 80.10$ & $€ 60.08$ \\
\hline Direct costs in depression & Veneto & Sardinia & & \\
\hline Total examination costs & $€ 157.35$ & $€|4| .70$ & & \\
\hline Hospitalization with attempted suicide & $€ 5,287.00$ & $€ 2,729.00$ & & \\
\hline Hospitalization without attempted suicide & $€ 3,403.50$ & $€ 1,962.00$ & & \\
\hline Cost of GP visit & $€ 12.00$ & $€ 12.00$ & & \\
\hline Number of GP visits/year & 7.50 & 7.50 & & \\
\hline Cost of first specialist visit & $€ 19.70$ & $€ 17.04$ & & \\
\hline Cost of follow-up specialist visit & $€ 13.70$ & $€ 11.36$ & & \\
\hline Number of specialist visits/year & 13.67 & 13.67 & & \\
\hline
\end{tabular}

Abbreviations: DDD, defined daily dose; MD, mean dose; GP, general practitioner; XR, extended-release.

scenario analysis was performed calculating monthly costs according to the mean dose (mean of minimum and maximum ranges taken from the summary of the product characteristics). These costs are shown in Table 5. For each drug prescription, a copayment (ticket) paid directly by the patient, when applicable, was subtracted from the respective prices.

\section{Utilities}

The TLV model, starting from Sobocki et al, ${ }^{24}$ adopted a utility value of 0.81 for a patient who achieves remission and a utility value of 0.57 for a patient who does not achieve remission. Moreover, it was assumed that utility values for patients who attempt suicide and for those in relapse were both equal to the utility value of patients who do not achieve remission (0.57). The expert panel did not confirm this

Table 6 Utilities

\begin{tabular}{lll}
\hline Utilities & Expert opinion & Sobocki et $\mathbf{~ a l}^{24}$ \\
\hline Patient in remission & 0.847 & 0.81 \\
Patient not in remission & 0.49 & 0.57 \\
Patient in relapse & 0.55 & - \\
Patient who attempts suicide & 0.267 & - \\
\hline
\end{tabular}

Notes: Data from Sobocki et al. ${ }^{24}$ Copyright (C) 2013. Dove Medical Press. Adapted with permission from Mencacci C, Di Sciascio G, Katz P, Ripellino C. Cost-effectiveness evaluation of escitalopram in major depressive disorder in Italy. Clinicoecon Outcomes Res. 2013;5:87-99. ${ }^{15}$ approach because, according to their clinical experience, these three disease stages have three different scores on the rating scales for depression. Based on this assumption, utility values were quantified by the expert panel, and these coefficients, identified "by consensus", were associated with the clinical status of patients (Table 6).

Nevertheless, the authors decided to test the results by performing a scenario analysis using the same utility values as those adopted in the TLV model.

\section{Analyses}

Cost utility analysis, used in this pharmacoeconomic evaluation, is a way of measuring the costs and benefits coming from a decision. This technique compares the costs of different procedures with their outcomes measured in "utility-based" units, ie, units that relate to a person's level of well-being. The most commonly used unit is the quality-adjusted life year (QALY). QALYs are calculated by estimating the total life years gained from a procedure and weighting each year to reflect the quality of life in that year. ${ }^{25}$

The incremental cost-effectiveness ratio (ICER) is the ratio of the estimated difference between the costs of two interventions and the estimated difference between the QALYs of these two interventions. It represents the estimated additional cost per extra unit of health generated by an 
intervention compared with its most cost-effective alternative for the same health condition.

Due to the lack of an official willingness-to-pay threshold in Italy, the authors decided to use an ICER threshold of $€ 25,000$ per QALY, which is slightly lower than the one recognized by the National Institute for Health and Clinical Excellence. ${ }^{26} \mathrm{We}$ decided to perform the following scenario analyses:

- mean dose calculated from the summary of the product characteristics

- utility values derived from Sobocki et al. ${ }^{24}$

We also performed a probabilistic sensitivity analysis using 10,000 Monte Carlo simulations. Gamma distributions were used for costs, except for treatment costs in which we applied deterministic costs, while betadistributions were applied for all probabilities and utilities of the model.

\section{Results}

\section{Base case analyses}

The results of the cost-effectiveness analysis are shown in Table 7. In the Veneto region, the SSRI escitalopram was associated with a lower total cost $(€ 1,604.9)$ compared with all the other treatment strategies, reflecting the fact that, on average, patients spend less time in the costly depression state. Further, escitalopram was associated with a larger health gain (QALYs) at one year (0.732), and therefore dominates the other treatment strategies because more QALYs are achieved at a lower total cost. In the Sardinia region, escitalopram was associated with a higher health gain (QALYs) at one year (0.732) compared with all the other treatment strategies whereas, from the cost side, escitalopram was associated

Table 7 Base case results

\begin{tabular}{lllll}
\hline Region & Strategy & Cost & Efficacy & ICER \\
\hline Veneto & Escitalopram & $€ I, 604.9$ & 0.732 & \\
& Venlafaxine XR & $€ I, 623.1$ & 0.729 & Dominated \\
& Sertraline & $€ I, 623.2$ & 0.724 & Dominated \\
& Paroxetine & $€ I, 64 I .9$ & 0.724 & Dominated \\
& Citalopram & $€ I, 670.7$ & 0.720 & Dominated \\
& Fluoxetine & $€ I, 675.6$ & 0.719 & Dominated \\
& Duloxetine & $€ I, 698.5$ & 0.727 & Dominated \\
& Fluvoxamine & $€ I, 922.7$ & 0.697 & Dominated \\
Sardinia & Sertraline & $€ I, I 22.0$ & 0.724 & \\
& Escitalopram & $€ I, I 38.1$ & 0.732 & $€ 2, I 20.5$ \\
& Paroxetine & $€ I, I 38.6$ & 0.724 & Dominated \\
& Venlafaxine XR & $€ I, I 4 I .2$ & 0.729 & Dominated \\
& Citalopram & $€ I, I 52.6$ & 0.720 & Dominated \\
& Fluoxetine & $€ I, I 55.6$ & 0.719 & Dominated \\
& Duloxetine & $€ I, 225.8$ & 0.727 & Dominated \\
& Fluvoxamine & $€ I, 3 I 3.0$ & 0.697 & Dominated \\
\hline
\end{tabular}

Abbreviations: ICER, incremental cost-effectiveness ratio; XR, extended-release. with a total cost of about $€ 1,138$, which was lower than for all the other treatment strategies, except for sertraline $(€ 1,122)$. Therefore, escitalopram dominates all the treatment strategies because more QALYs are achieved at a lower total cost except for sertraline, against which it was cost-effective, with an ICER of $€ 2,120.5$ per QALY gained.

Figure $2 \mathrm{~A}$ and $\mathrm{B}$ represent cost-effectiveness planes. Escitalopram was represented as the standard strategy because it was the most cost-effective strategy. These planes show that all the strategies are located in the third quadrant, meaning that they are dominated by escitalopram, except for the cost-effectiveness plane regarding the Sardinia region, in which sertraline is located in the fourth quadrant, meaning that it is less expensive but less effective.

\section{Alternative scenarios}

Two alternative scenarios have been tested: the first was the use of a different dose for calculating drug costs. We calculated the daily costs of the study drugs using the mean dose instead of the defined daily dosage used in the base case scenario. The results, shown in Table 8, confirmed those from base case with escitalopram dominating all the other antidepressants for Veneto. In Sardinia, sertraline was associated with a lower cost, followed by venlafaxine extended-release (XR), paroxetine, citalopram, fluoxetine, escitalopram, duloxetine, and fluvoxamine. Analyzing both the cost and effectiveness of the treatments, venlafaxine XR generated an ICER of about $€ 3,728$ per QALY gained compared with sertraline and escitalopram, which generated an ICER of about $€ 3,402$ per QALY gained compared with venlafaxine XR. All the other antidepressants were dominated.

The second tested scenario was adoption of the utilities derived from Sobocki et $\mathrm{al}^{24}$ used in the TLV model. This scenario analysis (Table 9) showed the dominance of escitalopram versus the other antidepressants, as also seen in the base case analysis for Veneto. Regarding Sardinia, as seen in the base case, escitalopram dominated all the other antidepressants except for sertraline, against which it was cost-effective, with an ICER of $€ 3,186.6$ per QALY gained.

\section{Probabilistic sensitivity analyses}

The cost-effectiveness acceptability curves are presented in Figure $3 \mathrm{~A}$ and B. For every value of willingness-to-pay considered, escitalopram is the most cost-effective strategy for Veneto. Given a threshold of $€ 25,000$ per QALY gained, there is around a $34 \%$ probability that escitalopram is cost-effective compared with the other treatments. Furthermore, compared with the other treatments, there is a $26 \%$ probability that escitalopram 


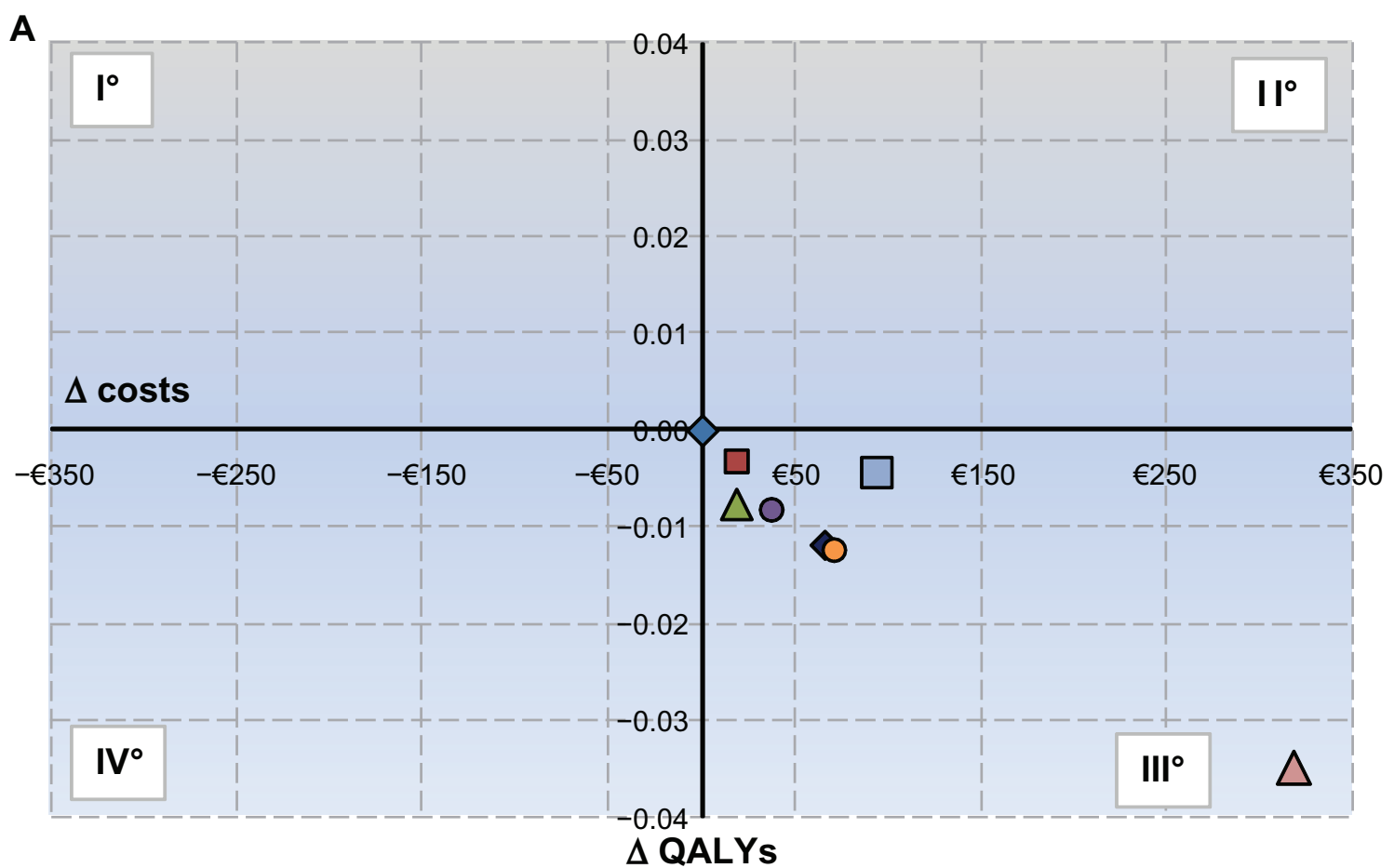
$\diamond$ Escitalopram
Venlafaxine XR
$\Delta$ Sertraline
O Paroxetine
$\diamond$ Citalopram
O Fluoxetine
口Duloxetine
$\Delta$ Fluvoxamine

B

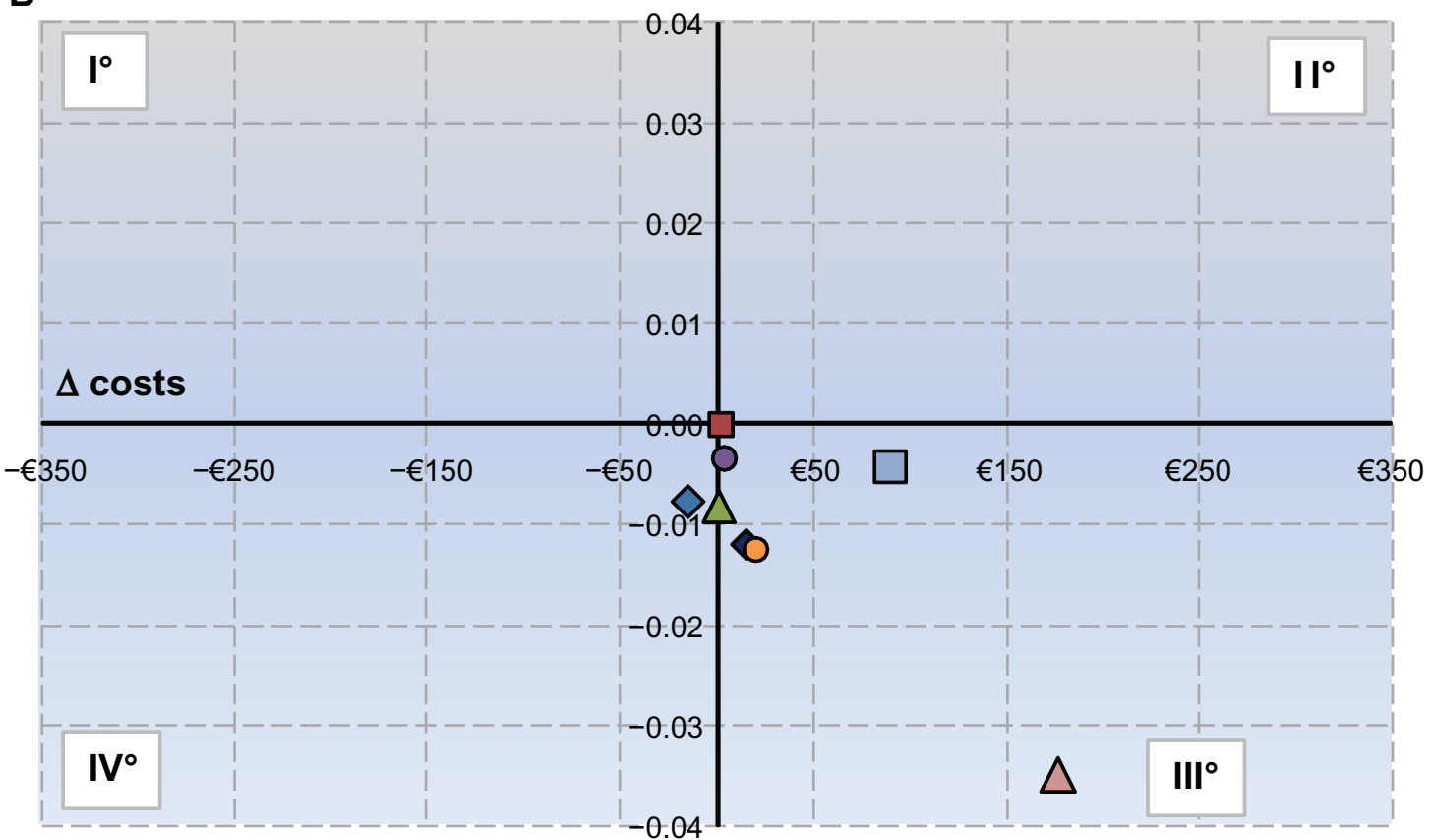

$\triangle$ QALYs

$\begin{array}{llll}\diamond \text { Sertraline } & \square \text { Escitalopram } & \Delta \text { Paroxetine } & \text { O Venlafaxine XR } \\ \diamond \text { Citalopram } & \text { O Fluoxetine } & \text { DDuloxetine } & \Delta \text { Fluvoxamine }\end{array}$

Figure 2 (A) Veneto cost-effectiveness plane. (B) Sardinia cost-effectiveness plane. Abbreviations: $X R$, extended-release; QALY, quality-adjusted life year. 
Table 8 Scenario analyses: use of mean dose

\begin{tabular}{lllll}
\hline Region & Strategy & Cost & Efficacy & ICER \\
\hline Veneto & Escitalopram & $€ I, 662.4$ & 0.732 & \\
& Venlafaxine XR & $€ I, 669.5$ & 0.729 & Dominated \\
& Sertraline & $€ I, 673.0$ & 0.724 & Dominated \\
& Paroxetine & $€ I, 693.3$ & 0.724 & Dominated \\
& Citalopram & $€ I, 7 I I .7$ & 0.720 & Dominated \\
& Fluoxetine & $€ I, 7 \mid 6.3$ & 0.719 & Dominated \\
& Duloxetine & $€ I, 776.4$ & 0.727 & Dominated \\
& Fluvoxamine & $€ I, 972.1$ & 0.697 & Dominated \\
Sardinia & Sertraline & $€ I, I 72.4$ & 0.724 & \\
& Venlafaxine XR & $€ I, I 89.0$ & 0.729 & $€ 3,728.9$ \\
& Paroxetine & $€ I, 190.6$ & 0.724 & Dominated \\
& Citalopram & $€ I, 194.4$ & 0.720 & Dominated \\
& Fluoxetine & $€ I, 197.1$ & 0.719 & Dominated \\
& Escitalopram & $€ I, \mid 198.3$ & 0.732 & $€ 3,402.2$ \\
& Duloxetine & $€ I, 3 \mid 2.1$ & 0.727 & Dominated \\
& Fluvoxamine & $€ I, 363.2$ & 0.697 & Dominated \\
\hline
\end{tabular}

Abbreviations: ICER, incremental cost-effectiveness ratio; $X R$, extended-release.

is cost-effective at a willingness-to-pay equal to zero, meaning that it is dominant in comparison with the other treatments.

For the Sardinia health services, escitalopram was the most cost-effective antidepressant for a willingness-to-pay of more than $€ 3,000$. Considering a threshold of $€ 25,000$ per QALY gained, there is an around $34 \%$ probability that escitalopram is costeffective compared with the other treatments. Nevertheless, at a willingness-to-pay equal to zero, escitalopram showed a $16 \%$ probability of being cost-effective dominating the other molecules.

\section{Discussion}

The C-QUALITY Project is the first cost-effectiveness analysis that compares all SSRIs and SNRIs in the first-line

Table 9 Scenario analyses: use of different utilities

\begin{tabular}{lllll}
\hline Region & Strategy & Cost & Efficacy & ICER \\
\hline Veneto & Escitalopram & $€ I, 604.9$ & 0.733 & \\
& Venlafaxine XR & $€ I, 623.1$ & 0.731 & Dominated \\
& Sertraline & $€ I, 623.2$ & 0.728 & Dominated \\
& Paroxetine & $€ I, 64 I .9$ & 0.727 & Dominated \\
& Citalopram & $€ I, 670.7$ & 0.725 & Dominated \\
& Fluoxetine & $€ I, 675.6$ & 0.725 & Dominated \\
& Duloxetine & $€ I, 698.5$ & 0.730 & Dominated \\
& Fluvoxamine & $€ I, 922.7$ & 0.710 & Dominated \\
Sardinia & Sertraline & $€ I, \mid 22.0$ & 0.728 & \\
& Escitalopram & $€ I, I 38.1$ & 0.733 & $€ 3, I 86.6$ \\
& Paroxetine & $€ I, I 38.6$ & 0.727 & Dominated \\
& Venlafaxine XR & $€ I, I 4 I .2$ & $0.73 \mid$ & Dominated \\
& Citalopram & $€ I, I 52.6$ & 0.725 & Dominated \\
& Fluoxetine & $€ I, I 55.6$ & 0.725 & Dominated \\
& Duloxetine & $€ I, 225.8$ & 0.730 & Dominated \\
& Fluvoxamine & $€ I, 3 \mid 3.0$ & 0.710 & Dominated \\
\hline
\end{tabular}

Abbreviations: ICER, incremental cost-effectiveness ratio; $X R$, extended-release. treatment of MDD in Italy and in a regional setting. The results for the Veneto model showed that in comparison with the seven antidepressants considered, escitalopram was less costly and more effective in terms of QALYs, dominating all the other pharmacologic treatments used in first line. The results from the Sardinia model showed that escitalopram dominated all the other pharmacologic treatments except for sertraline, against which it was cost-effective, generating an ICER of $€ 2,120.5$ per QALY gained; this value is much lower than the $€ 25,000$ threshold. Probabilistic sensitivity analyses and scenario simulations confirmed these results, indicating the robustness of the model.

Many other analyses ${ }^{14,27-30}$ fit with the results of this model. In a review of the use of antidepressants conducted by the TLV group, Wessling et al ${ }^{14}$ compared the costs and outcomes for first-line treatment with some of the most used antidepressants; in this health economics model for 12 months of treatment in Sweden, the results showed that escitalopram was the treatment giving the highest number of QALYs for the lowest cost (except for mirtazapine). All the other treatments were dominated by escitalopram. A study by Nordström et $\mathrm{al}^{27}$ compared the cost-effectiveness of escitalopram versus generic venlafaxine XR over a 6-month time frame for patients with MDD in Sweden. Compared with generic venlafaxine $\mathrm{XR}$, escitalopram was found to be less costly and more effective in terms of QALYs.

Ramsberg et $a^{28}$ developed a model to analyze the costeffectiveness of ten antidepressants. As in our study, remission rates were retrieved from a meta-analysis. The perspectives used were the societal and the health care service ones, while the time horizon was 12 months. The results showed that the most cost-effective pharmacologic treatment from a societal perspective was escitalopram, dominating all the other comparators, while from a health care perspective, the cost per QALY of escitalopram was $€ 3,732$ compared with that of venlafaxine.

The cost-effectiveness analysis presented by Nuijten et $\mathrm{a}^{29}$ compared venlafaxine XR, citalopram, and escitalopram from the societal perspective in a time horizon fixed at 26 weeks. The study results showed that escitalopram was associated with a mean cost saving of $€ 263$ per patient versus venlafaxine $X R$ and $€ 1,992$ versus citalopram. Escitalopram was also associated with a gain in QALYs of 0.0062 when compared with venlafaxine XR and of 0.0166 if compared with citalopram. Escitalopram was therefore dominant over both venlafaxine XR and citalopram.

Another cost-effectiveness analysis presented by Druais et al, ${ }^{30}$ also adapting the TLV model, compared eight 

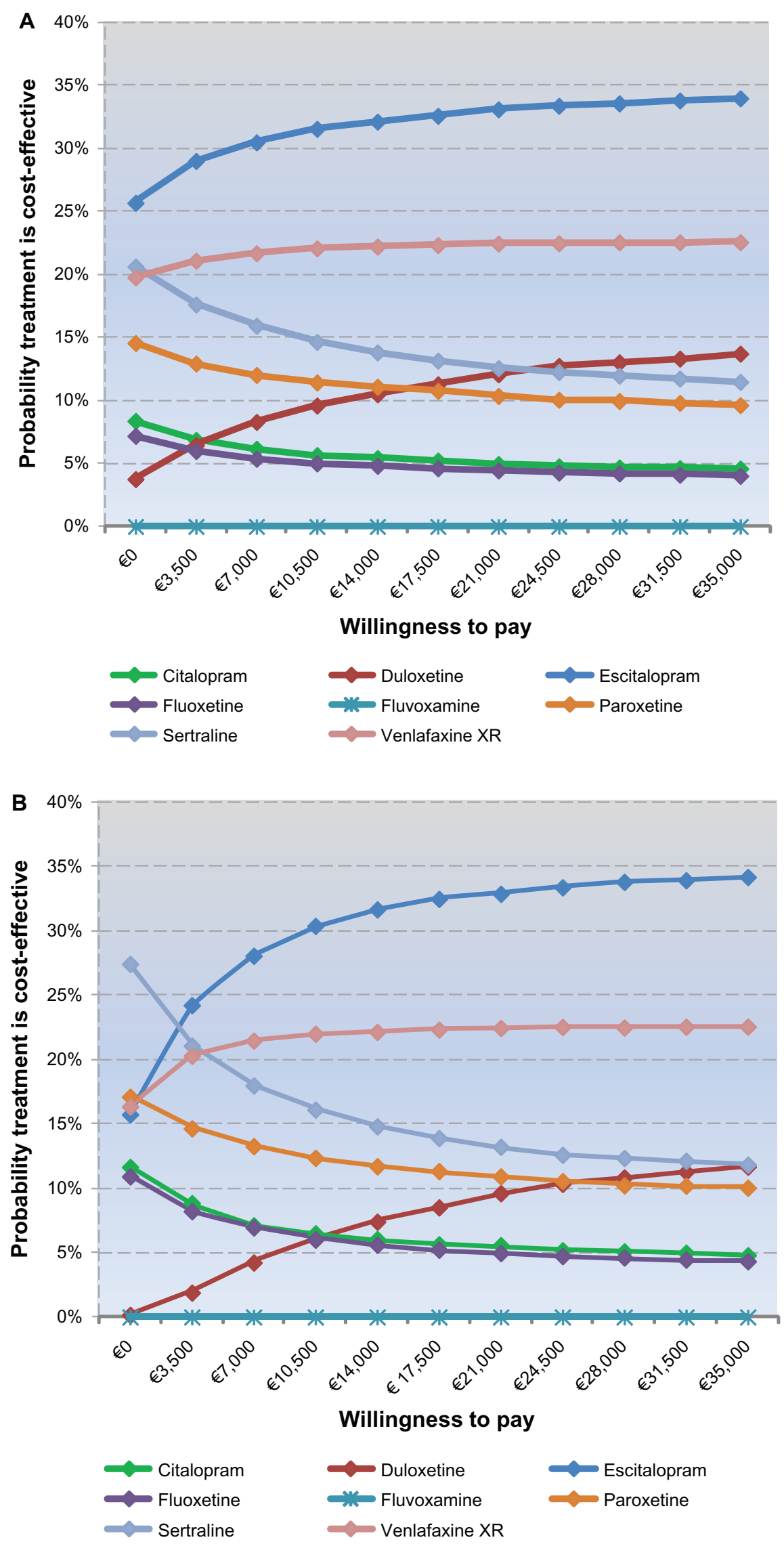

Figure 3 (A) Veneto cost-effectiveness acceptability curves. (B) Sardinia cost-effectiveness acceptability curves. Abbreviation: $X R$, extended-release. 
antidepressants from the perspectives of both society and the health care service, with a time horizon of one year and studying patients with MDD treated with a first-line antidepressant. The results showed that from the health care perspective escitalopram dominated all the other treatments except for venlafaxine, against which it was cost-effective, with an ICER of $€ 6,351$ per QALY gained; from the societal perspective, escitalopram dominated all the other strategies.

Several limitations to this study should be noted. For example, the main clinical inputs for the model, based on studies not performed in an Italian health care setting could be a limitation; nevertheless, the probabilities of clinical events are not usually considered to be country-specific. ${ }^{29}$ Another limitation regards the information about resource utilization, utilities, and treatment lines, that were estimated by the expert panel due to the lack of published local data. However, in situations in which the published material is insufficient or when literature review is unreliable, it has been suggested that use of expert opinion is considered appropriate. ${ }^{31}$ Further, sensitivity analyses with available published data have been performed, confirming the robustness of the model.

\section{Conclusion}

The presented cost-effectiveness analysis indicates that escitalopram is a cost-effective pharmacologic treatment strategy regarding the Veneto and Sardinia health services compared with the other SSRIs and SNRIs used in the first-line treatment of MDD. This is due to its lower direct costs compared with other treatment strategies and its higher effectiveness in terms of QALY gains.

\section{Author contributions}

PK and CR were responsible for model development and writing of the manuscript. CM, EA, GB, LC, GDS, AF, GM, and AT were responsible for clinical validation of the model and review of the manuscript. All authors read and approved the final manuscript.

\section{Disclosure}

This study was financially supported by an unrestricted grant from Lundbeck Italy $\mathrm{SpA}$, which was not responsible for creation of the study documents, the data analysis, data interpretation, or writing of the manuscript. CM, EA, GB, LC, GDS, AF, GM, and AT have received honoraria from CSD Medical Research Srl. PK and CR are employees of CSD Medical Research Srl. AF has received research grants and/or is/has been a consultant and/or a speaker for Angelini, Astra Zeneca, Bristol-Myers Squibb, Boehringer Ingelheim, Pfizer, Eli Lilly, Janssen, Lundbeck, Novartis, Otsuka, Roche, Sigma Tau, and Takeda. AT has been a speaker for Angelini, Astra Zeneca, Bristol-Myers Squibb, Boehringer Ingelheim, Pfizer, Eli Lilly, Janssen, Lundbeck, and Otsuka. Otherwise, the authors report no conflicts of interest in this work.

\section{References}

1. Belmaker RH, Agam G. Major depressive disorder. $N$ Engl J Med. 2008;358(1):55-68.

2. Mathers C, Boerma T, Ma Fat D. The Global Burden of Disease: 2004 Update. Geneva Switzerland: World Health Organization, 2008. Available from: http://www.who.int/healthinfo/global_burden_disease/ GBD_report_2004update_full.pdf. Accessed September 25, 2013.

3. Colombo G, Di Matteo S. [Budget impact analysis in the treatment of major depressive disorder on Italian regions: the role of venlafaxine]. Milan, Italy: WHERE Medical Press Srl; 2009. [Italian].

4. Battaglia A, Dubini A, Mannheimer R, Pancheri P. Depression in the Italian community: epidemiology and socio-economic implications. Int Clin Psychopharmacol. 2004;19(3):135-142.

5. Greenberg PE, Kessler RC, Birnbaum HG, et al. The economic burden of depression in the United States: how did it change between 1990 and 2000? J Clin Psychiatry. 2003;64(12):1465-1475.

6. Stoudemire A, Frank R, Hedemark N, Kamlet M, Blazer D. The economic burden of depression. Gen Hosp Psychiatry. 1986;8(6):387-394.

7. Frank L, Revicki DA, Sorensen SV, et al. The economics of selective serotonin reuptake inhibitors in depression: a critical review. CNS Drugs. 2001;15(1):59-83.

8. Séguin M, Lesage A, Chawky N, et al. Suicide cases in New Brunswick from April 2002 to May 2003: the importance of better recognizing substance and mood disorder comorbidity. Can J Psychiatry. 2006;51(9): 581-586.

9. Rapaport MH, Clary C, Fayyad R, Endicott J. Quality-of-life impairment in depressive and anxiety disorders. Am J Psychiatry. 2005;162(6): 1171-1178.

10. Panzarino PJ, Nash DB. Cost-effective treatment of depression with selective serotonin reuptake inhibitors. Am J Manag Care. 2001;7(2): 173-184.

11. Cipriani A, Furukawa TA, Salanti G, et al. Comparative efficacy and acceptability of 12 new-generation antidepressants: a multipletreatments meta-analysis. Lancet. 2009;373(9665):746-758.

12. Cipriani A, Santilli C, Furukawa TA, et al. Escitalopram versus other antidepressive agents for depression. Cochrane Database Syst Rev. 2009;2:CD006532.

13. Aguglia E, Ravasio R, Simonetti M, Pecchioli S, Mazzoleni F. Treatment modalities for SSRI and SNRI in Italy in 2003-2009. Curr Med Res Opin. 2012;28(9):1-10.

14. Wessling A, Ramsberg J. Depression: the review of antidepressants. Solna, Sweden: The Dental and Pharmaceutical Benefits Agency; 2008. Available from: http://www.tlv.se/Upload/genomgangen/reviewantidepressants.pdf. Accessed September 25, 2013.

15. Mencacci C, Di Sciascio G, Katz P, Ripellino C. Cost-effectiveness evaluation of escitalopram in major depressive disorder in Italy. Clinicoecon Outcomes Res. 2013;5:87-99.

16. Gaynes B, Rush A, Madhukar T, Wisniewski S, Spencer D, Fava M. The STAR*D study: treating depression in the real world. Cleve Clin J Med. 2008;75(1):57-66.

17. Hamilton M. A rating scale for depression. J Neurol Neurosurg Psychiatry. 1960;23:56-62.

18. Montgomery SA, Asberg M. A new depression scale designed to be sensitive to change. The British Journal of Psychiatry. 1979;134:382-389.

19. Gilchrist G, Gunn J. Observational studies of depression in primary care: what do we know? BMC Fam Pract. 2007;8:28. 
20. Lothgren M, Hemels M, Francois C, et al. Cost-effectiveness analysis of escitalopram as first line treatment of depression in Sweden. Prim Care Psychiatry. 2004;9(4):153-162.

21. Khan A, Khan SR, Leventhal RM, Brown WA. Symptom reduction and suicide risk in patients treated with placebo in antidepressant clinical trials: a replication analysis of the Food and Drug Administration Database. Int J Neuropsychopharmacol. 2001;4(2):113-118.

22. Agenzia Italiana del Farmaco. Rome: Agenzia Italiana del Farmaco. Available from: http://www.agenziafarmaco.gov.it/. Accessed November 21, 2012.

23. World Health Organization Collaborating Center. International language for drug utilization research. Oslo, Norway: World Health Organization Collaborating Center for Drug Statistics Methodology. Available from: http://www.whocc.no/. Accessed November 21, 2012.

24. Sobocki P, Ekman M, Agren H, Runeson B, Jonsson B. The mission is remission: health economic consequences of achieving full remission with antidepressant treatment for depression. Int J Clin Pract. 2006;60(7):791-798

25. Robinson R. Cost-utility analysis. BMJ. 1993;307:85924.

26. National Guideline Clearinghouse: Depression. The treatment and management of depression in adults. Guideline summary. London, UK: National Institute for Health and Clinical Excellence; 2009. Available from: http://guideline.gov/content.aspx?id=15521. Accessed December 17, 2012.
27. Nordström G, Danchenko N, Despiegel N, et al. Cost-effectiveness evaluation in Sweden of escitalopram compared with venlafaxine extended-release as first-line treatment in major depressive disorder. Value Health. 2012;15(2):231-239.

28. Ramsberg J, Asseburg C, Henriksson M. Effectiveness and costeffectiveness of antidepressants in primary care: a multiple treatment comparison meta-analysis and cost-effectiveness model. PLoS One. 2012;7(8):e42003.

29. Nuijten M, Brignone M, Marteau F, et al. Cost-effectiveness of escitalopram in major depressive disorder in Dutch health care. Clin Ther. 2012;34(6):1364-1378.

30. Druais S, Gauthier A, Demyttenaeare K, et al. An assessment of the cost-effectiveness of escitalopram vs multiple comparators as first-line antidepressant in patients with major depressive disorder (MDD) in Belgium. Presented at the International Society for Pharmacoeconomics and Outcomes Research Congress, Berlin, Germany, November 3-7, 2012.

31. Evans C. The use of consensus methods and expert panels in pharmacoeconomic studies: practical applications and methodological shortcomings. Pharmacoeconomics. 1997;12(2 Pt 1):121-129.
ClinicoEconomics and Outcomes Research

\section{Publish your work in this journal}

ClinicoEconomics \& Outcomes Research is an international, peerreviewed open-access journal focusing on Health Technology Assessment, Pharmacoeconomics and Outcomes Research in the areas of diagnosis, medical devices, and clinical, surgical and pharmacological intervention. The economic impact of health policy and health systems

\section{Dovepress}

organization also constitute important areas of coverage. The manuscript management system is completely online and includes a very quick and fair peer-review system, which is all easy to use. Visit http://www.dovepress.com/testimonials.php to read real quotes from published authors. 